\title{
Hybrid Dynamic Network Data Envelopment Analysis
}

\author{
Ling $\mathrm{Li}^{1}$ and Fengshan Wang ${ }^{2}$ \\ ${ }^{1}$ National Key Laboratory of Environmental Electromagnetic Effects and Electro-Optic Engineering, College of Field Engineering, \\ PLA University of Science and Technology, Nanjing 210007, China \\ ${ }^{2}$ College of Field Engineering, PLA University of Science and Technology, Nanjing 210007, China
}

Correspondence should be addressed to Fengshan Wang; wfs919@126.com

Received 16 December 2014; Accepted 9 April 2015

Academic Editor: Aura Reggiani

Copyright (C) 2015 L. Li and F. Wang. This is an open access article distributed under the Creative Commons Attribution License, which permits unrestricted use, distribution, and reproduction in any medium, provided the original work is properly cited.

Conventional DEA models make no hypothesis concerning the internal operations in a static situation. To open the "black box" and work with dynamic assessment issues synchronously, we put forward a hybrid model for evaluating the relative efficiencies of a set of DMUs over an observed time period with a composite of network DEA and dynamic DEA. We vertically deal with intermediate products between divisions with assignable inputs in the network structure and, horizontally, we extend network structure by means of a dynamic pattern with unrelated activities between two succeeding periods. The hybrid dynamic network DEA model proposed in this paper enables us to (i) pry into the internal operations of DEA by another network structure, (ii) obtain dynamic change of period efficiency, and (iii) gain the overall dynamic efficiency of DMUs over the entire observed periods. We finally illustrate the calculation procedure of the proposed approach by a numerical example.

\section{Introduction}

Data envelopment analysis (DEA), originated by Charnes, Cooper, and Rhodes, is a nonparametric approach for measuring the relative efficiency of homogeneous decision making units (DMUs), which use similar inputs to produce similar outputs [1]. DEA evaluates the efficiency of each DMU relative to an estimated production possibility frontier constructed by all DMUs. Traditional DEA approach is based on the thinking of "black box," which requires no assumption on the appearance of the frontier surface and it makes no hypothesis concerning the internal operations of a DMU. However, the actual transformation process is generally not modeled explicitly.

Network DEA allows us to look into these boxes and to evaluate organizational performance and its component performance (see, e.g., [2-5]). The development of network DEA model has come through four phases as follows: Firstly, Färe et al. [6] studied allocation of farmland to various crops, which allows for allocation of a (fixed) factor or input among alternative uses. This general structure could also be used to introduce allocation of a budget or allocation of resources across units or branches. This is the rudiment of network DEA model. Secondly, Löthgren and Tambour [7] proposed a kind of network model that allows inclusion of customer satisfaction in efficiency and productivity measures. The network consists of a production node and a consumption node and offers flexibility in modelling the production and consumption process where a firm-specific allocation of input resources to production and customer oriented activities is allowed. In this phase, intermediate products were emphasized. Thirdly, Färe and Grosskopf [8] suggested dynamic DEA model which considered time factor. The dynamic DEA model introduced here is used to study the dynamic efficiency of APEC (Asian-Pacific Economic Community) countries. The network formulation is a dynamic DEA model in which some outputs at period $t$ are inputs in the next period, $t+1$. However, the third phase ignores the structure of network itself which is discussed in the second phase. Thus, it is a simple network model with time parameter, but not real dynamic network DEA model. Fourthly, Tone and Tsutsui [9] dealt with intermediate products formally to evaluate divisional efficiencies along with the overall efficiency of DMUs by a slack-based measure. 
They handled any network structure in which nodes were connected and intermediates were directed.

Measurement of intertemporal efficiency change has long been an interesting subject of concern in DEA (called dynamic DEA). The dynamic DEA was explored to assess DMUs performance from a long-term perspective using carry-over variables. The dynamic DEA originated by Färe et al. [6] is the first innovative algorithm for dealing with interconnecting activities. It was further developed by several authors from different perspectives. In recent years, Chen and van Dalen [10] developed a dynamic DEA that incorporates dynamic factors via a linear parametric formulation. Soleimani-damaneh [11] provided a recursive enumeration algorithm for solving the nonconvex dynamic DEA models. Soleimani-damaneh [12] also utilized the envelopment models for estimating returns to scale in dynamic DEA. To cope with long time point of view, Tone and Tsutsui [13] put forward a dynamic DEA model that adds carry-over activities into the model to measure period specific efficiency based on the long time optimization during the whole period. Then, Tone and Tsutsui extended the model by a combination of network DEA and dynamic DEA [14]. However, sub-DMUs of their hybrid dynamic network DEA were connected by links by means of chain network structure. The intermediate products between sub-DMUs were neglected and inputs cannot be allocated to them.

Hence, this paper is a hybrid composite of dynamic and network DEA. However, our dynamic network DEA model deals with network structure with intermediate products between any sub-DMUs and copes with assignable inputs over observed dynamic periods. The hybrid model proposed in this paper enables us to open the "black box" to reveal the internal connections of DMUs as well as realizing dynamic evaluation of network DEA over the entire observed periods. The rest of this paper unfolds as follows. In Section 2, we formulate network DEA model with intermediates and assignable inputs. In Section 3, we propose a hybrid model called dynamic network DEA (DNDEA), combining dynamic DEA with network DEA. We also construct a new DEA unit to obtain the dynamic efficiency of network DEA. In Section 4, we exhibit an illustrative example to explain the operational process of the hybrid model. Some conclusions are presented in Section 5.

\section{Network DEA Model}

2.1. Introduction of DEA. DEA is a widely used operational programming approach for comparing the multiple inputs and outputs of a set of homogenous DMUs by measuring their relative efficiency. Suppose that there are $n$ DMUs to be evaluated where each $\mathrm{DMU}_{j}, j=1,2, \ldots, n$, consumes $m$ inputs, $x_{i j}(i=1,2, \ldots, m)$, producing $s$ outputs, $y_{r j}(r=$ $1,2, \ldots, s)$. The structure of conventional DEA can be viewed as a "black box" described in Figure 1.

The CCR (Charnes, Cooper, and Rhodes) model for evaluating the technical input-efficiency of $j_{0}$ th DMU $\left(\mathrm{DMU}_{0}\right)$ under constant returns to scale (CRS) is represented as

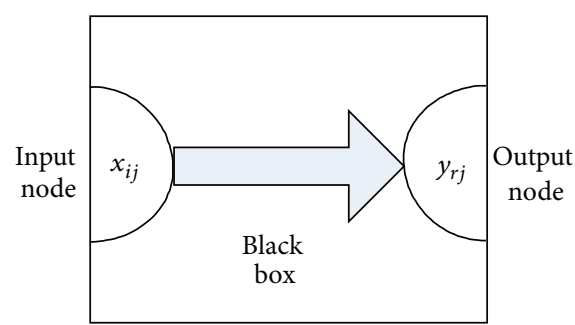

Figure 1: The structure of conventional DEA.

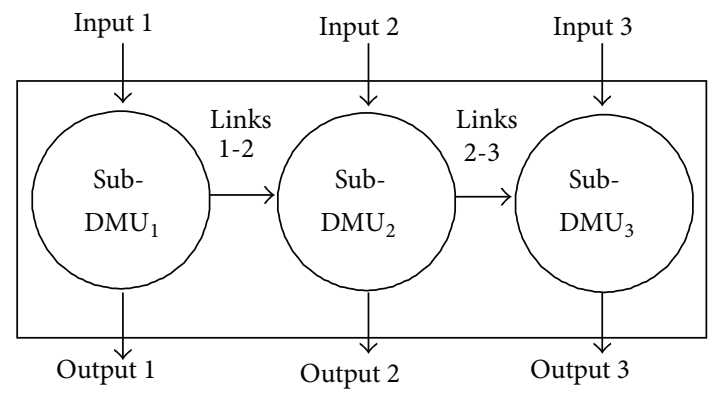

FIGURE 2: The structure of network DEA with chain links and nonassignable inputs.

$$
P_{\mathrm{CCR}}:\left\{\begin{array}{l}
\max h_{0}=\sum_{r=1}^{s} \mu_{r} y_{r 0} \\
\sum_{i=1}^{m} \omega_{i} x_{i 0}=1 \\
\sum_{i=1}^{m} \omega_{i} x_{i j}-\sum_{r=1}^{s} \mu_{r} y_{r j} \geq 0 ; j=1,2, \ldots, n \\
\omega_{i}, \mu_{r} \geq 0, \forall i, r .
\end{array}\right.
$$

As an extension of the "black box" model above, Färe and Grosskopf [8] introduced the network DEA model with chain links and nonassignable inputs illustrated in Figure 2. Tone and Tsutsui [13] developed the network structure by applying it in the dynamic network DEA using a slack-based measure.

2.2. Network DEA with Intermediate Products. In this paper, we propose a network structure with intermediate products between any two sub-DMUs. And the inputs can be assigned to sub-DMUs as well. We suppose that there are $n$ homogeneous DMUs which involve $s$ sub-DMUs $P_{l}(l=$ $1,2, \ldots, s)$. The input and output of $\mathrm{DMU}_{j}$ are $X_{j}^{0}$ and $Y_{j}^{e}$, respectively. Inputs of sub-DMUs received from input node are $X_{j}^{0 l}$ and $X_{j}^{0 r}$, which are assignable, and outputs of subDMUs exported to output node are $Y_{j}^{l e}$ and $Y_{j}^{r e}$. There are input and output values between sub-DMUs which we call intermediate products; $\widetilde{X}_{j}^{r l}$ is defined as input from $P_{r}$ to $P_{l}$; $\widetilde{Y}_{j}^{l r}$ is defined as output from $P_{l}$ to $P_{r}$, where $r, l \in\{1,2, \ldots, s\}$, $r \neq l$. We can deduce that $\widetilde{X}_{j}^{r l}=\widetilde{Y}_{j}^{r l}$ and $\widetilde{X}_{j}^{l r}=\widetilde{Y}_{j}^{l r}$. Figure 3 illustrates the structure of network DEA with intermediate products and assignable inputs. 


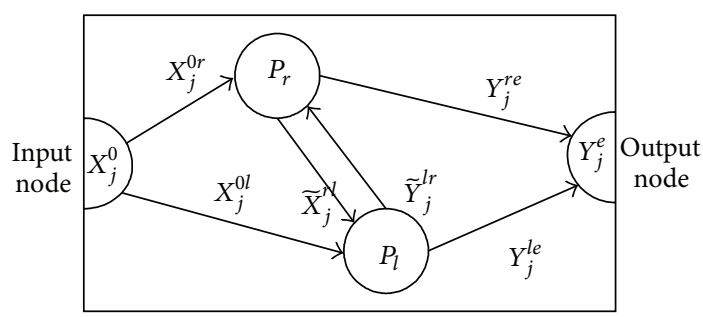

FIgURE 3: The structure of network DEA with intermediate products and assignable inputs.

When we take intermediate products into consideration in DEA model, we can formulate network DEA as follows:

$$
\begin{aligned}
& \left(P_{\text {Network }}\right): \\
& \left\{\begin{array}{l}
\max \left(\mu^{e T} Y_{0}^{e}\right)=V_{P} \\
\omega^{0 l T} X_{j}^{0 l}+\sum_{r=1, r \neq l}^{s} \widetilde{\omega}^{r l T} \widetilde{X}_{j}^{r l}-\mu^{l e T} Y_{j}^{l e}-\sum_{r=1, r \neq l}^{s} \widetilde{\mu}^{l r T} \widetilde{Y}_{j}^{l r} \geq 0, \\
j=1,2, \ldots, n ; l=1,2, \ldots, s ; \omega^{0 T} X_{0}^{0}=1, \omega^{0} \geq \omega^{0 l} \\
\widetilde{\mu}^{r l} \geq \widetilde{\omega}^{r l}, \omega^{0 l} \geq 0, \widetilde{\omega}^{r l} \geq 0, \mu^{e} \geq 0, \mu^{l e} \geq 0, \widetilde{\mu}^{r l} \geq 0 .
\end{array}\right.
\end{aligned}
$$

The dual model of network DEA above can be transformed as (3), where $\theta$ is the network DEA efficiency of $\mathrm{DMU}_{0}$, which reflects the overall efficiency of network DEA with intermediate products. $\theta$ is proportional to the performance of the evaluated DMU:

$$
\left(D_{\text {Network }}\right):\left\{\begin{array}{l}
\min \theta=V_{D} \\
\sum_{l=1}^{s} X_{0}^{0 l}=\theta X_{0}^{0}, \sum_{j=1}^{n} \lambda_{j}^{l} X_{j}^{0 l}+S^{0 l-}=X_{0}^{0 l}, \sum_{j=1}^{n} \lambda_{j}^{l} \widetilde{X}_{j}^{r l}+\widetilde{S}^{r l-}=\widetilde{X}_{0}^{r l} \\
\sum_{j=1}^{n} \lambda_{j}^{l} Y_{j}^{l e}-S^{l e+}=Y_{0}^{l e}, \sum_{j=1}^{n} \lambda_{j}^{l} \widetilde{Y}_{j}^{l r}-\widetilde{S}^{l r+}=\widetilde{Y}_{0}^{l r}, l, r \in\{1,2, \ldots, s\}, l \neq r \\
\lambda_{j} \geq 0, j=1,2, \ldots, n ; X_{0}^{0 l} \geq 0, S^{0 l-} \geq 0, \widetilde{S}^{r l-} \geq 0, S^{l e+} \geq 0, \widetilde{S}^{l r+} \geq 0 .
\end{array}\right.
$$

\section{Hybrid Dynamic Network DEA}

3.1. Dynamic Network DEA Model. Network DEA has the structure with intermediate products between input node and output node, which shows a network form. However, it is a static modality essentially. On the basis of decision making space of network DEA, we introduce the time parameter $t$ to formulate three-dimensional decision making issue, called dynamic network DEA (DN-DEA). During the $t$ th time span, the input of $\mathrm{DMU}_{j}$ is $X_{j(t)}^{0}$ and the output of $\mathrm{DMU}_{j}$ is $Y_{j(t)}^{e}$, which can be formulated as

$$
\begin{aligned}
X_{j(t)}^{0}= & \left(x_{1(t)}^{0}, x_{2(t)}^{0}, \ldots, x_{n(t)}^{0}\right) \\
= & \left(\begin{array}{ccccc}
x_{11(t)}^{0} & \cdots & x_{1 j(t)}^{0} & \cdots & x_{1 n(t)}^{0} \\
\cdots & \cdots & \cdots & \cdots & \cdots \\
x_{i 1(t)}^{0} & \cdots & x_{i j(t)}^{0} & \cdots & x_{i n(t)}^{0} \\
\cdots & \cdots & \cdots & \cdots & \cdots \\
x_{m 1(t)}^{0} & \cdots & x_{m j(t)}^{0} & \cdots & x_{m n(t)}^{0}
\end{array}\right)_{m \times n},
\end{aligned}
$$

$$
\begin{aligned}
Y_{j(t)}^{e}= & \left(y_{1(t)}^{e}, y_{2(t)}^{e}, \ldots, y_{n(t)}^{e}\right) \\
= & \left(\begin{array}{ccccc}
y_{11(t)}^{e} & \cdots & y_{1 j(t)}^{e} & \cdots & y_{1 n(t)}^{e} \\
\cdots & \cdots & \cdots & \cdots & \cdots \\
y_{k 1(t)}^{e} & \cdots & y_{k j(t)}^{e} & \cdots & y_{k n(t)}^{e} \\
\cdots & \cdots & \cdots & \cdots & \cdots \\
y_{s 1(t)}^{e} & \cdots & y_{s j(t)}^{e} & \cdots & y_{s n(t)}^{e}
\end{array}\right)_{s \times n}
\end{aligned}
$$

Inputs of sub-DMUs received from input node are $X_{j(t)}^{0 l}$ and $X_{j(t)}^{0 r}$, and outputs of sub-DMUs exported to output node are $Y_{j(t)}^{l e}$ and $Y_{j(t)}^{r e}$. There are input and output values between subDMUs in the $t$ th time span; $\widetilde{X}_{j(t)}^{r l}$ is defined as input from $P_{r}$ to $P_{l} ; \widetilde{Y}_{j(t)}^{l r}$ is defined as output from $P_{l}$ to $P_{r}$, where $r, l \in$ $\{1,2, \ldots, s\}, r \neq l, t=1,2, \ldots, T$. We can also deduce that $\widetilde{X}_{j(t)}^{r l}=\widetilde{Y}_{j(t)}^{r l}$ and $\widetilde{X}_{j(t)}^{l r}=\widetilde{Y}_{j(t)}^{l r}$. We can describe the structure of DN-DEA in the $t$ th time span as in Figure 4.

The DN-DEA model can be formed as follows:

$$
\left(P_{\mathrm{DN}}\right):\left\{\begin{array}{l}
\max \left(\mu_{(t)}^{e T} Y_{0(t)}^{e}\right)=V_{P(t)} \\
\omega_{(t)}^{0 l T} X_{j(t)}^{0 l}+\sum_{r=1, r \neq l}^{s} \widetilde{\omega}_{(t)}^{r l T} \widetilde{X}_{j(t)}^{r l}-\mu_{(t)}^{l e T} Y_{j(t)}^{l e}-\sum_{r=1, r \neq l}^{s} \widetilde{\mu}_{(t)}^{l r T} \widetilde{Y}_{j(t)}^{l r} \geq 0, \\
j=1,2, \ldots, n ; l=1,2, \ldots, s ; t=1,2, \ldots, T, \omega_{(t)}^{0 T} X_{0(t)}^{0}=1, \omega_{(t)}^{0} \geq \omega_{(t)}^{0 l} \\
\tilde{\mu}_{(t)}^{r l} \geq \widetilde{\omega}_{(t)}^{r l}, \omega_{(t)}^{0 l} \geq 0, \widetilde{\omega}_{(t)}^{r l} \geq 0, \mu_{(t)}^{e} \geq 0, \mu_{(t)}^{l e} \geq 0, \widetilde{\mu}_{(t)}^{r l} \geq 0 .
\end{array}\right.
$$




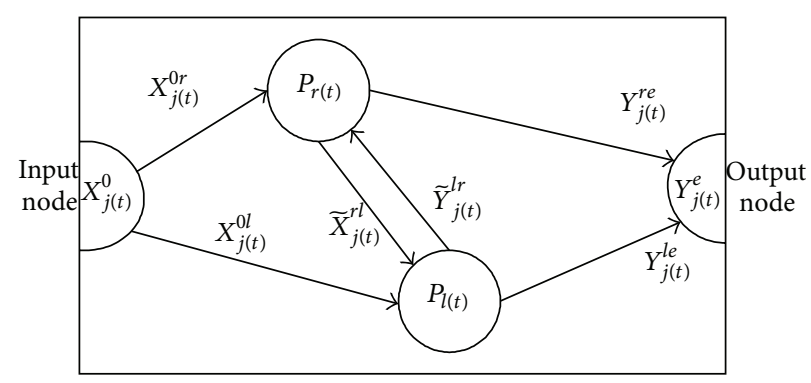

Figure 4: The structure of DN-DEA in one time span.

The dual model of the above formulation can be transformed as (6), where $\theta^{(t)}$ is the network DEA efficiency of $\mathrm{DMU}_{0}$ in the $t$ th time span $(t=1,2, \ldots, T)$, showing dynamic efficiency of DN-DEA:

$$
\left(D_{\mathrm{DN}}\right):\left\{\begin{array}{l}
\min \theta^{(t)}=V_{D(t)} \\
\sum_{l=1}^{s} X_{0(t)}^{0 l}=\theta^{(t)} X_{0(t)}^{0} \\
\sum_{j=1}^{n} \lambda_{j(t)}^{l} X_{j(t)}^{0 l}+S_{(t)}^{0 l-}=X_{0(t)}^{0 l}, \sum_{j=1}^{n} \lambda_{j(t)}^{l} \widetilde{X}_{j(t)}^{r l}+\widetilde{S}_{(t)}^{r l}=\widetilde{X}_{0(t)}^{r l} \\
\sum_{j=1}^{n} \lambda_{j(t)}^{l} Y_{j(t)}^{l e}-S_{(t)}^{l e+}=Y_{0(t)}^{l e}, \sum_{j=1}^{n} \lambda_{j(t)}^{l} \widetilde{Y}_{j(t)}^{l r}-\widetilde{S}_{(t)}^{l r+}=\widetilde{Y}_{0}^{l r}, \\
l, r \in\{1,2, \ldots, s\}, l \neq r ; \lambda_{j(t)} \geq 0, j=1,2, \ldots, n ; t=1,2, \ldots, T, \\
X_{0(t)}^{0 l} \geq 0, S_{(t)}^{0 l-} \geq 0, \widetilde{S}_{(t)}^{r l-} \geq 0, S_{(t)}^{l e+} \geq 0, \widetilde{S}_{(t)}^{l r+} \geq 0 .
\end{array}\right.
$$

3.2. Dynamic Evaluation of DN-DEA Efficiencies. $\theta_{j}^{(t)}$ is the DN-DEA efficiency of $\mathrm{DMU}_{j}$ in the th time span. We define $\theta_{j}^{(t)}$ as the DN-DEA efficiency matrix, which means efficiencies of DN-DEA model in discrete time spans. The DN-DEA efficiency matrix can be formulated as follows:

$$
\begin{aligned}
\theta_{j}^{(t)} & =\left(\theta_{1}^{(t)}, \theta_{2}^{(t)}, \ldots, \theta_{n}^{(t)}\right) \\
& =\left(\begin{array}{ccccc}
\theta_{1}^{1} & \cdots & \theta_{j}^{1} & \cdots & \theta_{n}^{1} \\
\cdots & \cdots & \cdots & \cdots & \cdots \\
\theta_{1}^{t} & \cdots & \theta_{j}^{t} & \cdots & \theta_{n}^{t} \\
\cdots & \cdots & \cdots & \cdots & \cdots \\
\theta_{1}^{T} & \cdots & \theta_{j}^{T} & \cdots & \theta_{n}^{T}
\end{array}\right)_{T \times n} .
\end{aligned}
$$

The column vector of $\theta_{j}^{(t)}$ reflects the dynamic efficiency of DN-DEA over the whole period of each DMU. Hence, measuring the dynamic network efficiency of DN-DEA is a typical multiple attributes decision making issue essentially. In addition to DEA model, many scholars have put forward some mathematical approaches to solve the problem, for example, analytic hierarchy process, grey correlation analysis, and fuzzy comprehensive evaluation, which have their own advantages and disadvantages. In this work, we apply DEA approach for the "second round" on the basis of hybrid DNDEA model formulated above. In view of the structure of
DN-DEA efficiency matrix to be evaluated, $\theta_{j}^{(t)}$ represents the network DEA efficiency of $\mathrm{DMU}_{j}$ in the $t$ th time span. However, the matrix itself has no network structure inside. Thus, we can build a new "black box" DEA to assess overall dynamic efficiency for DN-DEA. For DEA model, input and output index have their normal requirements: input is inversely proportional to the DEA efficiency; output is proportional to the DEA efficiency. From the analysis above we know that $\theta_{j}^{(t)}$ is proportional to the performance of $\mathrm{DMU}_{j}$ in period $t$; thus we can treat it as output of the new DEA. Moreover, input can be formed as $1 \times n$ dimensional vector. In order to operate easily, we treat it as $1 \times n$ dimensional unit vector. The structure diagram of the new DEA is displayed as shown in Figure 5.

From the new constructed DEA as shown in Figure 5, we can build the dynamic evaluation model of DN-DEA efficiency, which can be formulated as follows:

$$
\left(D^{I} \varepsilon\right):\left\{\begin{array}{l}
\min \left[\sigma-\varepsilon\left(\hat{e}^{T} S^{-}+e^{T} S^{+}\right)\right]=\xi \\
\sum_{j=1}^{n} 1 \times \lambda_{j}+S^{-}=\sigma \times 1 \\
\sum_{j=1}^{n} \theta_{j}^{(t)} \lambda_{j}-S^{+}=\theta_{0}^{(t)} \\
\lambda_{j} \geq 0, j=1,2, \ldots, n ; S^{-} \geq 0, S^{+} \geq 0,
\end{array}\right.
$$




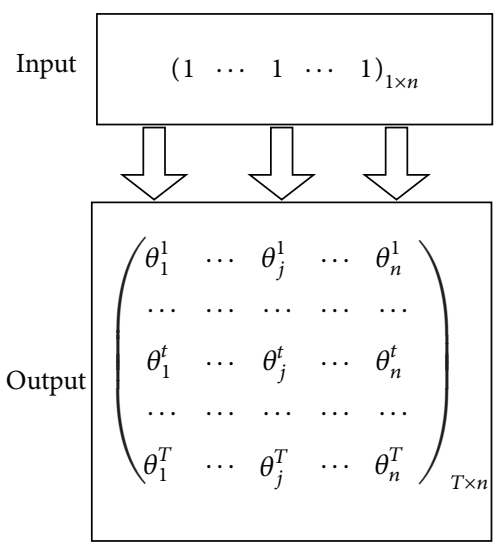

FIGURE 5: The constructed DEA for measuring dynamic efficiency of DN-DEA.

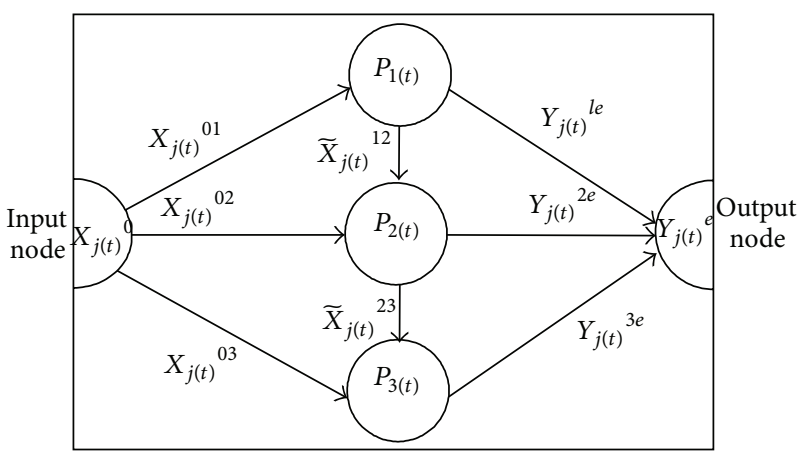

FIGURE 6: DN-DEA with 3 sub-DMUs in the one time span.

where $\widehat{e}=(1,1, \ldots, 1)^{T} \in E^{1}$ and $e=(1,1, \ldots, 1)^{T} \in E^{T}$ are unit vectors, $\varepsilon(\varepsilon>0)$ is a non-Archimedean infinitesimal, and $S^{-}$and $S^{+}$are slacks of DMUs. $\xi$ is the dynamic efficiency of $\mathrm{DMU}_{0}$ all over $T$ period in DN-DEA model. We can further transform the model to

$$
\left(D^{I} \varepsilon\right):\left\{\begin{array}{l}
\min \left[\sigma-\varepsilon\left(\hat{e}^{T} S^{-}+e^{T} S^{+}\right)\right]=\xi \\
\sum_{j=1}^{n} \lambda_{j}=\sigma-S^{-}, \sum_{j=1}^{n} \theta_{j}^{(t)} \lambda_{j}=\theta_{0}^{(t)}+S^{+} \\
\lambda_{j} \geq 0, j=1,2, \ldots, n, S^{-} \geq 0, S^{+} \geq 0 .
\end{array}\right.
$$

\section{Numerical Example}

In this section, a numerical example is presented to illustrate DN-DEA model. To this end, ten DMUs with one input node, one output node, and three sub-DMUs are considered over four time periods. We can describe the DN-DEA structure of this example in Figure 6. It is worth mentioning that there are intermediate products between sub-DMU $\mathrm{U}_{1}$ and sub-DMU and between sub- $\mathrm{DMU}_{2}$ and sub- $\mathrm{DMU}_{3}$. The data of inputs, outputs, and intermediate products for these ten DMUs over the observed four time periods have been listed in Tables 1-4.

By running (6), we can obtain the network DEA efficiency of ten DMUs in the $t$ th time span $(t=1,2,3,4)$, also

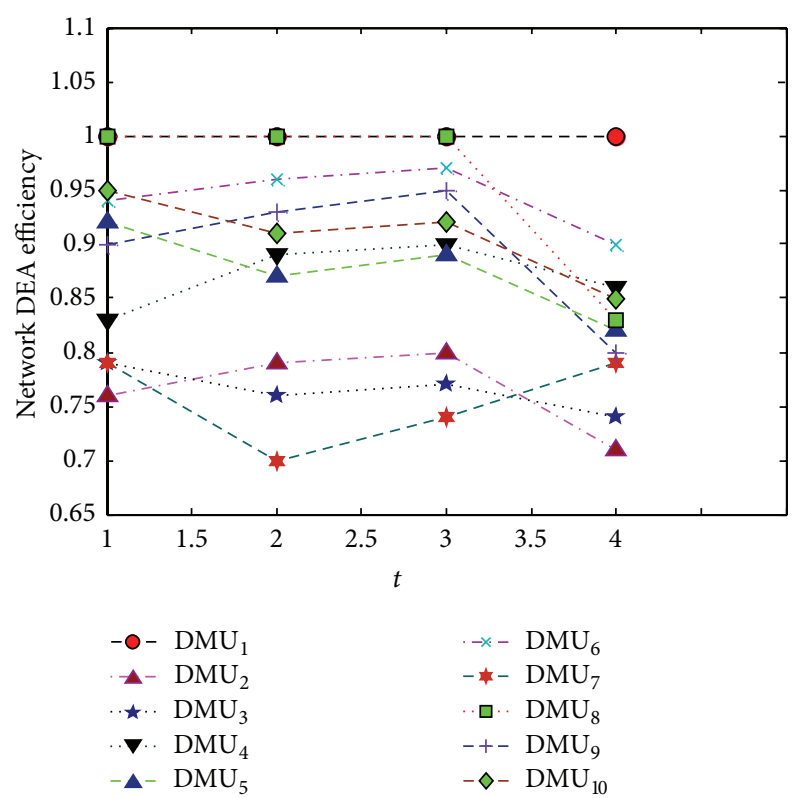

FIGURe 7: Dynamic fluctuation of network DEA efficiency.

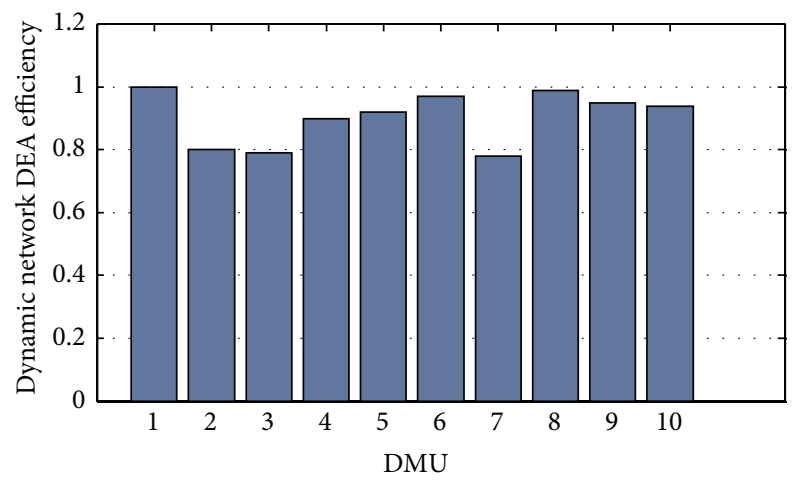

FIGURE 8: Dynamic network DEA efficiency of 10 DMUs.

summarized in Tables 1-4. Thus, we can gain DN-DEA efficiency matrix as shown in

$$
\begin{aligned}
& \theta_{j}^{(t)} \\
& =\left(\begin{array}{cccccccccc}
1 & 0.76 & 0.79 & 0.83 & 0.92 & 0.94 & 0.79 & 1 & 0.90 & 0.95 \\
1 & 0.79 & 0.76 & 0.89 & 0.87 & 0.96 & 0.70 & 1 & 0.93 & 0.91 \\
1 & 0.80 & 0.77 & 0.90 & 0.89 & 0.97 & 0.74 & 1 & 0.95 & 0.92 \\
1 & 0.71 & 0.74 & 0.86 & 0.82 & 0.90 & 0.79 & 0.83 & 0.80 & 0.85
\end{array}\right) .
\end{aligned}
$$

Figure 7 reflects the dynamic fluctuation of network DEA efficiency for each DMU over the four time periods, from which we can conclude that the network DEA efficiency of the observed time periods vibrates irregularly. We apply model (9) for the sake of evaluating the overall dynamic network efficiency of DN-DEA on the basis of the DEA structure proposed in Figure 5. The overall dynamic network efficiency and ranking sequence of DN-DEA can be finally provided in Table 5, which can be compared in Figure 8 . Table 5 also provides slacks from which we can get improved 
TABLE 1: Evaluation data of DN-DEA $(t=1)$.

\begin{tabular}{lcccccccccc}
\hline \multirow{2}{*}{ DMU $_{j}$} & Efficiency & Input & \multicolumn{3}{c}{ Sub-DMU } & \multicolumn{3}{c}{ Sub-DMU $\mathrm{U}_{2}$} & \multicolumn{2}{c}{ Sub-DMU } \\
& $\theta_{j}^{1}$ & $X_{j}^{0}$ & $X_{j}^{01}$ & $\widetilde{X}_{j}^{12}$ & $Y_{j}^{1 e}$ & $X_{j}^{02}$ & $\widetilde{X}_{j}^{23}$ & $Y_{j}^{2 e}$ & $X_{j}^{03}$ & $Y_{j}^{3 e}$ \\
\hline 1 & 1 & 4170 & 2412 & 4310 & 195 & 1066 & 722 & 198 & 692 & 910 \\
2 & 0.76 & 6349 & 4662 & 7710 & 191 & 963 & 414 & 189 & 724 \\
3 & 0.79 & 8046 & 6395 & 7939 & 183 & 936 & 510 & 183 & 715 & 510 \\
4 & 0.83 & 4679 & 2522 & 5533 & 188 & 1252 & 568 & 177 & 905 & 599 \\
5 & 0.92 & 5938 & 4211 & 5194 & 177 & 1012 & 621 & 199 & 715 & 814 \\
6 & 0.94 & 4442 & 3148 & 4426 & 195 & 891 & 204 & 193 & 403 & 355 \\
7 & 0.79 & 7036 & 5338 & 6623 & 185 & 956 & 493 & 187 & 742 & 493 \\
8 & 1 & 5122 & 3606 & 6490 & 194 & 906 & 681 & 196 & 610 & 858 \\
9 & 0.90 & 5740 & 4253 & 5285 & 185 & 775 & 289 & 192 & 712 & 355 \\
10 & 0.95 & 4680 & 3087 & 3839 & 189 & 780 & 580 & 193 & 813 & 630 \\
\hline
\end{tabular}

TABLE 2: Evaluation data of DN-DEA $(t=2)$.

\begin{tabular}{lcccccccccc}
\hline \multirow{2}{*}{$\mathrm{DMU}_{j}$} & Efficiency & Input & \multicolumn{3}{c}{ Sub-DMU } & \multicolumn{3}{c}{ Sub-DMU $\mathrm{DMb}_{2}$} & \multicolumn{2}{c}{ Sub-DMU $_{3}$} \\
& $\theta_{j}^{1}$ & $X_{j}^{0}$ & $X_{j}^{01}$ & $\widetilde{X}_{j}^{12}$ & $Y_{j}^{1 e}$ & $X_{j}^{02}$ & $\widetilde{X}_{j}^{23}$ & $Y_{j}^{2 e}$ & $X_{j}^{03}$ & $Y_{j}^{3 e}$ \\
\hline 1 & 1 & 3820 & 2308 & 4209 & 207 & 932 & 677 & 213 & 580 & 996 \\
2 & 0.79 & 6082 & 4564 & 7608 & 211 & 840 & 408 & 198 & 678 & 512 \\
3 & 0.76 & 8199 & 6432 & 7966 & 168 & 997 & 518 & 143 & 770 & 448 \\
4 & 0.89 & 4457 & 2443 & 5411 & 190 & 1130 & 550 & 187 & 884 & 625 \\
5 & 0.87 & 6144 & 4321 & 5233 & 141 & 1136 & 664 & 178 & 687 & 905 \\
6 & 0.96 & 4290 & 3028 & 4297 & 212 & 870 & 189 & 197 & 392 & 392 \\
7 & 0.70 & 8307 & 6408 & 6667 & 176 & 1102 & 535 & 168 & 797 & 445 \\
8 & 1 & 4929 & 3503 & 6370 & 209 & 886 & 656 & 208 & 540 & 967 \\
9 & 0.93 & 5583 & 4105 & 5123 & 194 & 770 & 248 & 197 & 708 & 377 \\
10 & 0.91 & 4867 & 3134 & 3899 & 170 & 884 & 598 & 168 & 849 \\
\hline
\end{tabular}

TABLE 3: Evaluation data of DN-DEA $(t=3)$.

\begin{tabular}{|c|c|c|c|c|c|c|c|c|c|c|}
\hline \multirow{2}{*}{$\mathrm{DMU}_{j}$} & \multirow{2}{*}{$\begin{array}{c}\text { Efficiency } \\
\theta_{j}^{1}\end{array}$} & \multirow{2}{*}{$\begin{array}{c}\text { Input } \\
X_{j}^{0}\end{array}$} & \multicolumn{3}{|c|}{ Sub-DMU } & \multicolumn{3}{|c|}{ Sub-DMU ${ }_{2}$} & \multicolumn{2}{|c|}{ Sub-DMU } \\
\hline & & & $X_{j}^{01}$ & $\widetilde{X}_{j}^{12}$ & $Y_{j}^{1 e}$ & $X_{j}^{02}$ & $\widetilde{X}_{j}^{23}$ & $Y_{j}^{2 e}$ & $X_{j}^{03}$ & $Y_{j}^{3 e}$ \\
\hline 1 & 1 & 3500 & 2150 & 4100 & 230 & 884 & 654 & 222 & 466 & 1033 \\
\hline 2 & 0.80 & 5762 & 4406 & 7499 & 234 & 792 & 385 & 207 & 564 & 549 \\
\hline 3 & 0.77 & 7879 & 6274 & 7857 & 191 & 949 & 495 & 152 & 656 & 485 \\
\hline 4 & 0.90 & 4137 & 2285 & 5302 & 213 & 1082 & 527 & 196 & 770 & 662 \\
\hline 5 & 0.89 & 5824 & 4163 & 5124 & 164 & 1088 & 641 & 187 & 573 & 942 \\
\hline 6 & 0.97 & 3970 & 2870 & 4188 & 235 & 822 & 166 & 206 & 278 & 429 \\
\hline 7 & 0.74 & 7987 & 6250 & 6558 & 199 & 1054 & 512 & 177 & 683 & 482 \\
\hline 8 & 1 & 4609 & 3345 & 6261 & 232 & 838 & 633 & 217 & 426 & 1004 \\
\hline 9 & 0.95 & 5263 & 3947 & 5014 & 217 & 722 & 225 & 206 & 594 & 414 \\
\hline 10 & 0.92 & 4547 & 2976 & 3790 & 193 & 836 & 575 & 177 & 735 & 665 \\
\hline
\end{tabular}

information between inefficient DMUs and optimum values by means of projection. We can know that $\mathrm{DMU}_{1}$ performs best and $\mathrm{DMU}_{7}$ displays worst. The ranking sequence shows that $\mathrm{DMU}_{1}>\mathrm{DMU}_{8}>\mathrm{DMU}_{6}>\mathrm{DMU}_{9}>\mathrm{DMU}_{10}>$ $\mathrm{DMU}_{5}>\mathrm{DMU}_{4}>\mathrm{DMU}_{2}>\mathrm{DMU}_{3}>\mathrm{DMU}_{7}$.

\section{Conclusions}

In this paper, we put forward a hybrid dynamic network DEA model, taking dynamic assessment and network structure in
DEA into consideration simultaneously. The hybrid system measures the relative efficiencies of a set of DMUs with network structure of intermediate products between subDMUs with assignable inputs over observed time periods. The dynamic efficiency reflects overall network efficiency during the same discrete time spans, showing more practical meaning. What is more, improved information between inefficient DMUs and optimum values can be provided to decision makers by means of non-Archimedean infinitesimal and slacks of DMUs. Notably, different dynamic structure of 
TABLE 4: Evaluation data of DN-DEA $(t=4)$.

\begin{tabular}{|c|c|c|c|c|c|c|c|c|c|c|}
\hline \multirow{2}{*}{$\mathrm{DMU}_{j}$} & \multirow{2}{*}{$\begin{array}{c}\text { Efficiency } \\
\theta_{j}^{1}\end{array}$} & \multirow{2}{*}{$\begin{array}{c}\text { Input } \\
X_{j}^{0}\end{array}$} & \multicolumn{3}{|c|}{ Sub-DMU } & \multicolumn{3}{|c|}{ Sub-DMU ${ }_{2}$} & \multicolumn{2}{|c|}{ Sub-DMU 3} \\
\hline & & & $X_{j}^{01}$ & $\widetilde{X}_{j}^{12}$ & $Y_{j}^{1 e}$ & $X_{j}^{02}$ & $\widetilde{X}_{j}^{23}$ & $Y_{j}^{2 e}$ & $X_{j}^{03}$ & $Y_{j}^{3 e}$ \\
\hline 1 & 1 & 4509 & 2531 & 4523 & 178 & 1202 & 809 & 183 & 776 & 947 \\
\hline 2 & 0.71 & 6688 & 4781 & 7923 & 174 & 1099 & 501 & 174 & 808 & 529 \\
\hline 3 & 0.74 & 8385 & 6514 & 8152 & 166 & 1072 & 597 & 168 & 799 & 547 \\
\hline 4 & 0.86 & 5018 & 2641 & 5746 & 171 & 1388 & 655 & 162 & 989 & 636 \\
\hline 5 & 0.82 & 6277 & 4330 & 5407 & 160 & 1148 & 708 & 184 & 799 & 851 \\
\hline 6 & 0.90 & 4781 & 3267 & 4639 & 178 & 1027 & 291 & 178 & 487 & 392 \\
\hline 7 & 0.79 & 7375 & 5457 & 6836 & 168 & 1092 & 580 & 172 & 826 & 530 \\
\hline 8 & 0.83 & 5461 & 3725 & 6703 & 177 & 1042 & 768 & 181 & 694 & 895 \\
\hline 9 & 0.80 & 6079 & 4372 & 5498 & 168 & 911 & 376 & 177 & 796 & 392 \\
\hline 10 & 0.85 & 5019 & 3206 & 4052 & 172 & 916 & 667 & 178 & 897 & 667 \\
\hline
\end{tabular}

TABLE 5: Evaluation results of DN-DEA.

\begin{tabular}{lccccccc}
\hline $\mathrm{DMU}_{j}$ & $\xi_{j}$ & $S_{1}^{-}$ & $S_{1}^{+}$ & $S_{2}^{+}$ & $S_{3}^{+}$ & $S_{4}^{+}$ & Ranking \\
\hline 1 & 1 & 0 & 0 & 0 & 0 & 0 & 1 \\
2 & 0.80 & 0 & 0.04 & 0.01 & 0 & 0.09 & 8 \\
3 & 0.79 & 0 & 0 & 0.03 & 0.02 & 0.05 & 9 \\
4 & 0.90 & 0 & 0.07 & 0.01 & 0 & 0.04 & 7 \\
5 & 0.92 & 0 & 0 & 0.05 & 0.03 & 0.10 & 6 \\
6 & 0.97 & 0 & 0.03 & 0.01 & 0 & 0.66 & 3 \\
7 & 0.78 & 0 & 0 & 0.09 & 0.05 & 0 & 10 \\
8 & 0.99 & 0 & 0 & 0 & 0 & 0.17 & 2 \\
9 & 0.95 & 0 & 0.05 & 0.02 & 0 & 0.15 & 4 \\
10 & 0.94 & 0 & 0 & 0.04 & 0.03 & 0.10 & 5 \\
\hline
\end{tabular}

network DEA such as carry-over dynamic type or continuous one may need diverse models for measuring corresponding dynamic efficiency of DN-DEA. The dynamic evaluation results of DN-DEA will depend on the constructed dynamic model related to the defined dynamic structure. The "black box" DEA model set up above adapts to the structure of DN-DEA efficiency matrix. However, other DEA models give more importance to more recent years. For instance, superefficiency DEA will help to solve unsatisfactory differentiation of overall dynamic network efficiencies. Further work based on the framework proposed in this paper could extend the hybrid dynamic network model to include other dynamic patterns as well as different network structures.

\section{Conflict of Interests}

The authors declare that there is no conflict of interests regarding the publication of this paper.

\section{Acknowledgments}

This work was supported by the National Natural Science Foundation of China (Grant no. 51308541), the Natural Science of Jiangsu Province (Grant no. BK20130066), and the Foundation of National Key Laboratory on Environmental Electromagnetic Effects and Electro-Optic Engineering (Grand no. FD2015008).

\section{References}

[1] A. Charnes, W. W. Cooper, and E. Rhodes, "Measuring the efficiency of decision making units," European Journal of Operational Research, vol. 2, no. 6, pp. 429-444, 1978.

[2] S. Lozano and E. Gutiérrez, "A slacks-based network DEA efficiency analysis of European airlines," Transportation Planning and Technology, vol. 37, no. 7, pp. 623-637, 2014.

[3] T.-S. Chang, K. Tone, and Q. Wei, "Ownership-specified network DEA models," Annals of Operations Research, vol. 214, pp. 73-98, 2014.

[4] M. Maghbouli, A. Amirteimoori, and S. Kordrostami, "Twostage network structures with undesirable outputs: a DEA based approach," Measurement, vol. 48, no. 1, pp. 109-118, 2014.

[5] M. Tavana, H. Mirzagoltabar, S. M. Mirhedayatian, R. F. Saen, and M. Azadi, "A new network epsilon-based DEA model for supply chain performance evaluation," Computers \& Industrial Engineering, vol. 66, no. 2, pp. 501-513, 2013.

[6] R. Färe, R. Grabowski, S. Grosskopf, and S. Kraft, "Efficiency of a fixed but allocatable input: a non-parametric approach," Economics Letters, vol. 56, no. 2, pp. 187-193, 1997.

[7] M. Löthgren and M. Tambour, "Productivity and customer satisfaction in Swedish pharmacies: a DEA network model," European Journal of Operational Research, vol. 115, no. 3, pp. 449-458, 1999.

[8] R. Färe and S. Grosskopf, "Network DEA," Socio-Economic Planning Sciences, vol. 34, no. 1, pp. 35-49, 2000.

[9] K. Tone and M. Tsutsui, "Network DEA: a slacks-based measure approach," European Journal of Operational Research, vol. 197, no. 1, pp. 243-252, 2009.

[10] C.-M. Chen and J. van Dalen, "Measuring dynamic efficiency: theories and an integrated methodology," European Journal of Operational Research, vol. 203, no. 3, pp. 749-760, 2010.

[11] M. Soleimani-damaneh, "An enumerative algorithm for solving nonconvex dynamic DEA models," Optimization Letters, vol. 7, no. 1, pp. 101-115, 2013.

[12] M. Soleimani-damaneh, "Another approach for estimating RTS in dynamic DEA," Journal of Productivity Analysis, vol. 39, no. 1, pp. 75-81, 2013. 
[13] K. Tone and M. Tsutsui, "Dynamic DEA: a slacks-based measure approach," Omega, vol. 38, no. 3-4, pp. 145-156, 2010.

[14] K. Tone and M. Tsutsui, "Dynamic DEA with network structure: a slacks-based measure approach," Omega, vol. 42, no. 1, pp. 124131, 2014. 


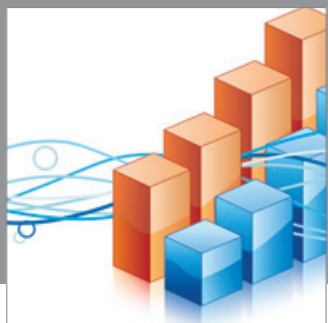

Advances in

Operations Research

mansans

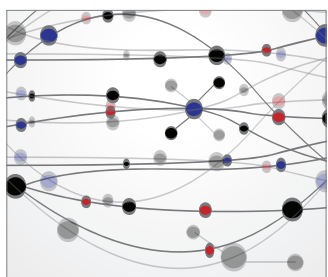

The Scientific World Journal
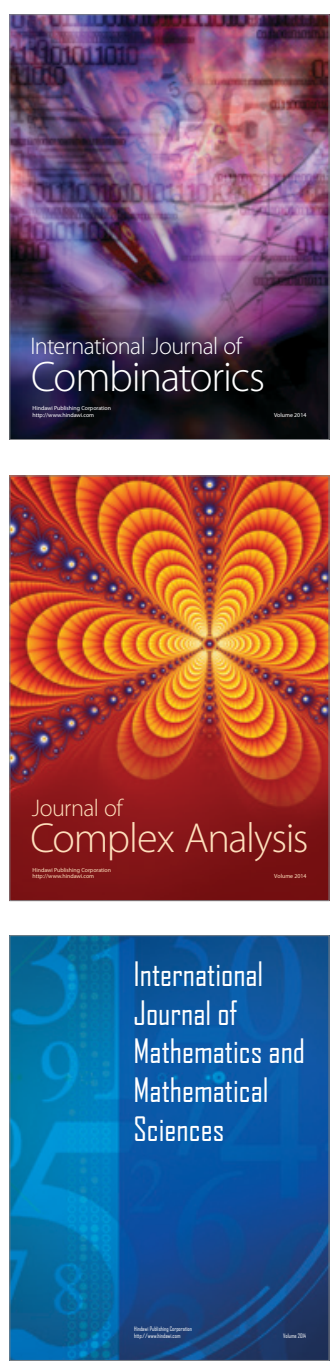
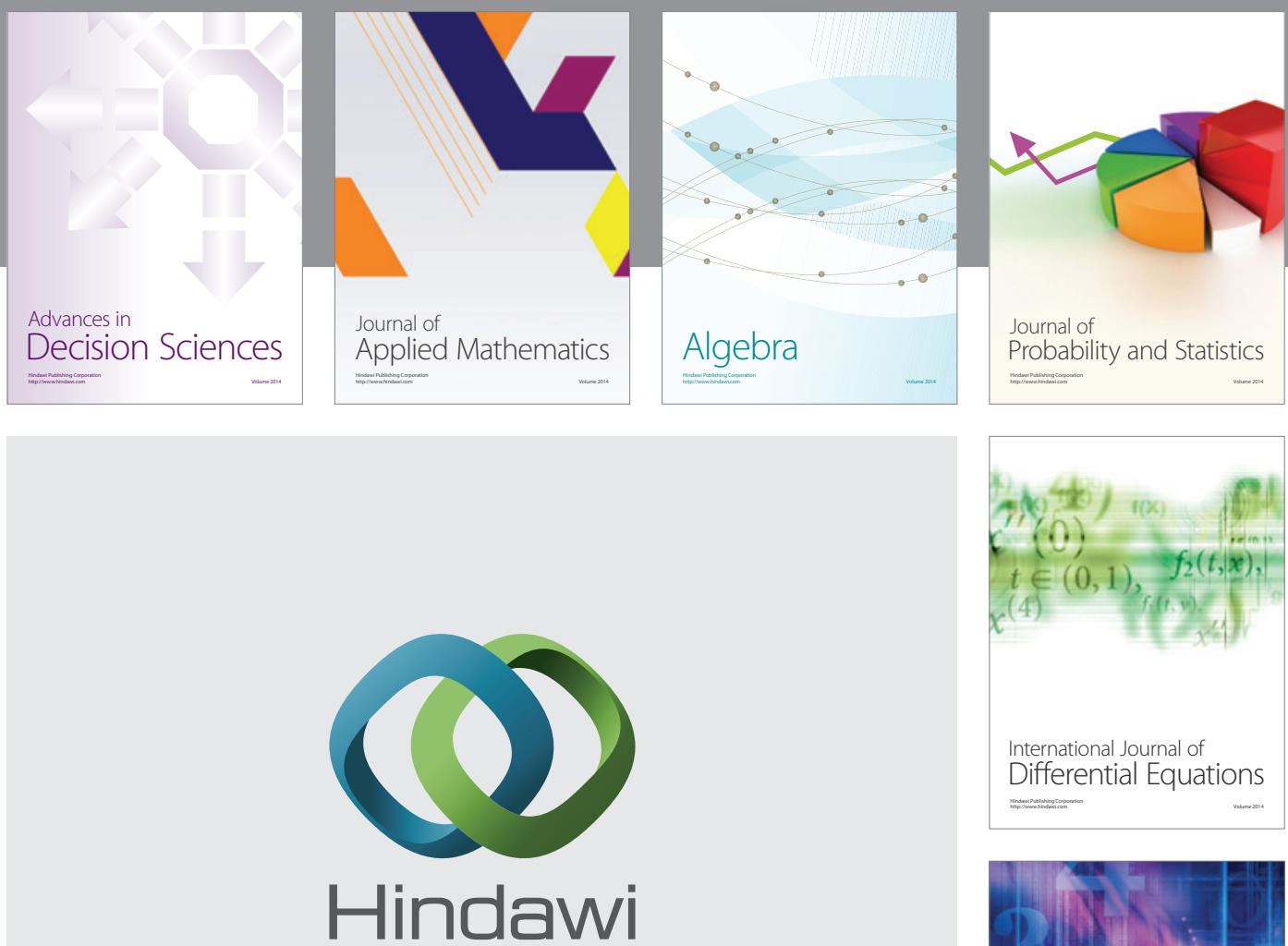

Submit your manuscripts at http://www.hindawi.com
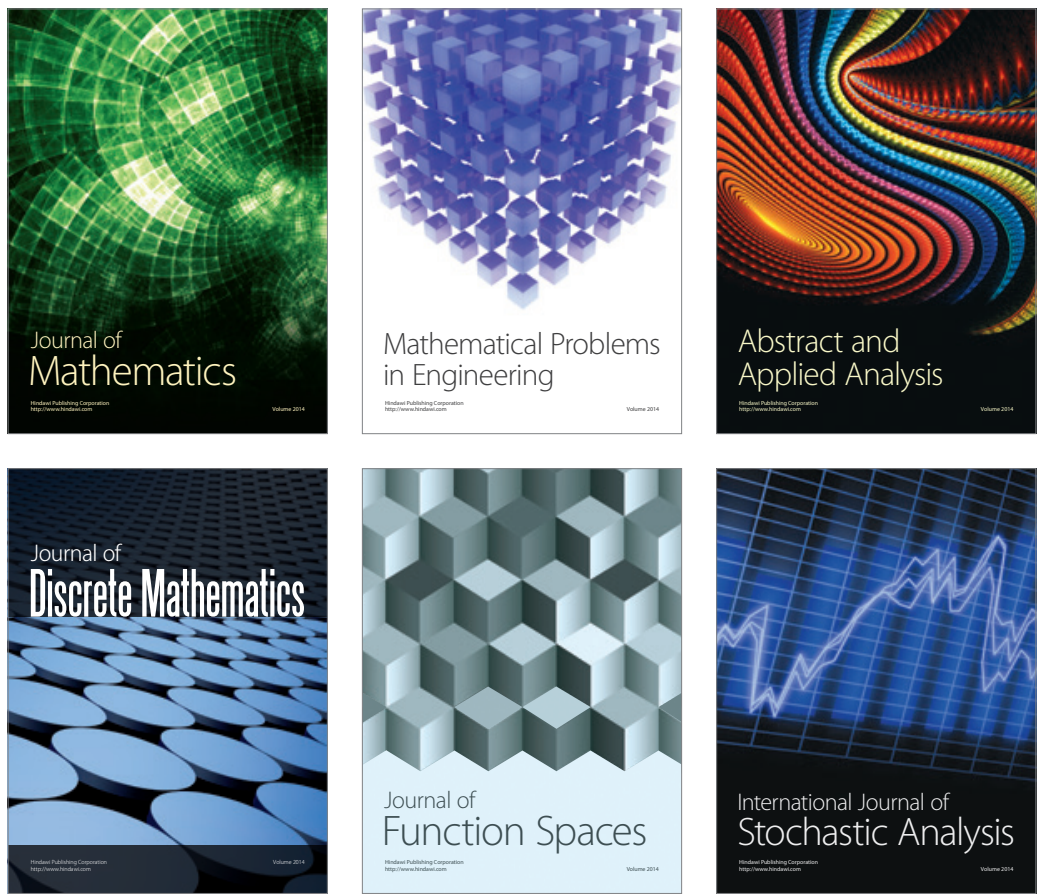

Journal of

Function Spaces

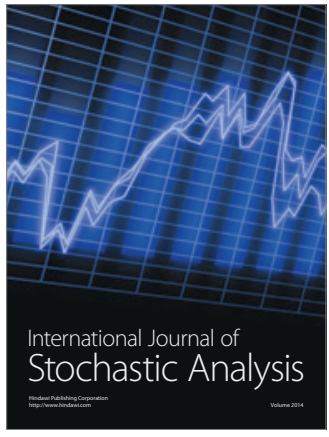

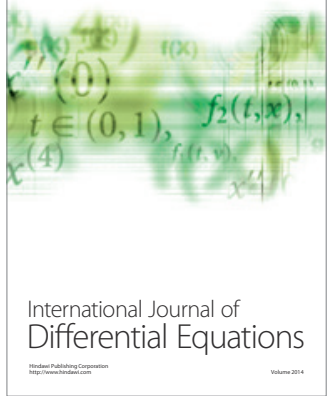
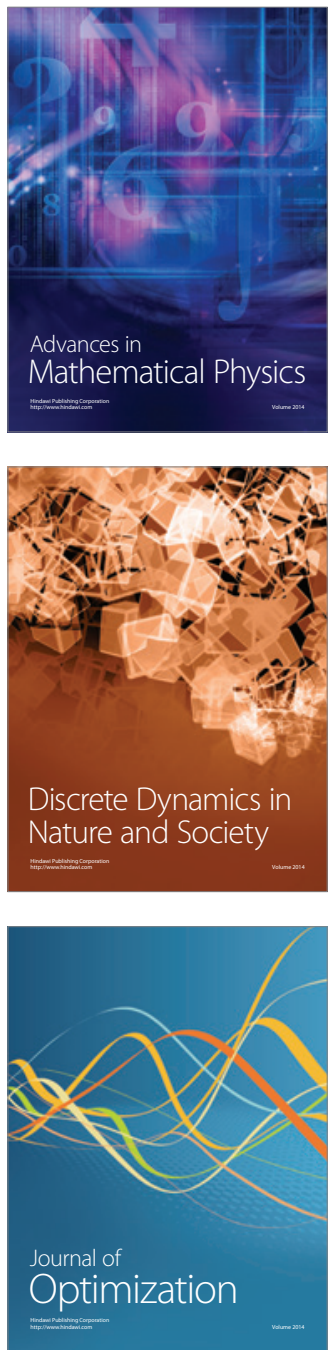